\title{
FORMULAÇÃO DE BOLOS ENRIQUECIDOS COM FARINHAS PRODUZIDAS COM SUBPRODUTOS DA LARANJA
}

\section{FORMULATION OF ENRICHED CAKES WITH ORANGE BY-PRODUCTS}

\author{
Marianela Díaz Urrutia ${ }^{1 *}$, Amanda Gemelli Ramos ${ }^{1}$, Marina Horst ${ }^{1}$, Tássia Sacks ${ }^{1}$, \\ Bruna Bedin ${ }^{1}$, Daniela Miotto Bernardi ${ }^{2}$ \\ 1Discente do curso de Nutrição do Centro Universitário Fundação Assis Gurgacz (FAG). ${ }^{2}$ Nutricionista \\ 'Doutora, Docente do Curso de Nutrição, Centro Universitário Fundação Assis Gurgacz (FAG). \\ *Autor correspondente: marianeladiazurrutia@gmail.com, https://orcid.org/0000-0001-5047-953X
}

\section{RESUMO}

A maior parte da produção total de laranja no Brasil é destinada à indústria de suco, gerando subprodutos ricos em bioativos, que normalmente são descartados. Nesse contexto, o trabalho teve por objetivo desenvolver e avaliar sensorialmente, formulações de bolos fortificadas com farinhas a base de subprodutos da laranja. Assim, foram produzidas duas farinhas, uma de casca de laranja e outra de casca e bagaço de laranja, onde, a matéria prima de ambas, foi previamente desidratada em estufa de circulação e logo triturada até formar as respectivas farinhas. Posteriormente foram desenvolvidas três formulações de bolos: Formulação 1 (F1): bolo tradicional de laranja, Formulação 2 (F2): bolo de aveia fortificado com farinha de casca de laranja, Formulação 3 (F3): bolo de aveia fortificado com farinha de casca e bagaço de laranja. A análise sensorial mostrou que, F1 teve uma aceitação significativamente melhor $(p<0,05)$ para todos os parâmetros avaliados, do que F2 e F3 que não diferiram estatisticamente $(p>0,05)$ entre si. Tanto a aveia como a casca de laranja, apresentam características funcionais que podem enriquecer preparações nutricionalmente, porém a palatabilidade de preparações com ingredientes tradicionais se reflete em uma melhor aceitação nos consumidores.

Palavras-chave: polifenóis; limoneno; bioativos.

\begin{abstract}
The most amount of total oranges production in Brazil is for juice industry, creating subproducts that are rich in bioactive, that generally aren't used. In this context, this job had the goal of develop and evaluate formules of cakes fortificated with flours made from subproducts of orange. So two kind of flours were produced. One of them was made from orange's husk and the other one was made from the husk and mark of the oranges. The raw material of both was previously dehydrated in an oven of circulation and then it was crushed until to get both kind of flours. Later there were developed three formules of cakes: F1: traditional orange cake; F2: oat cake fortified with flour made from husk and mark of the orange. The sensory analysis showed that F1 had a higher significant acceptance $(p<0,05)$ for all the parameters evaluated, than F2 and F3 that didn't have statistical difference among them. The oat and the husk of orange have functional features that could enrich nutritional preparations. Although the palatability of preparations with traditional ingredients is seen in a better acceptation from consumers.
\end{abstract}

Key words: polyphenols; limonene; bioactives. 


\section{INTRODUÇÃO}

Nos últimos anos, o Brasil vem se destacando no comercio mundial de laranjas como o maior produtor desta fruta, pois o baixo custo de produção favorece o plantio em grande escala no país (FRANCO, 2016).

Considera-se que, a maior parte da produção total de laranja no Brasil é destinada, principalmente, à indústria de suco e uma parte menor destina-se à elaboração de outros produtos, tais como: doce em calda, doce em massa, fruta cristalizada, pectina, fermentado alcoólico, fermentado acético e aguardente (LIMA et al., 2010).

Segundo dados fornecidos pela United States Department of Agriculture (USDA), o Ministério da Agricultura do Brasil (2018), afirma que no período 2016/2017, o Brasil realizou um aporte de $66,4 \%$ na produção global de suco de laranja. Posicionando o nosso país como o principal produtor e exportador mundial deste tipo de suco.

Para Cypriano (2017), durante a transformação da laranja em suco, $50 \%$ da fruta é descartada como casca e bagaço, sendo ricos em bioativos, onde se destaca a presença de óleos essenciais, principalmente o D-limoneno (FERNANDES et al., 2013), fibra alimentar (SANTANA; GASPARETTO, 2009) e polifenóis (STORCK, 2013). A EMBRAPA (2001), afirma que uma parte destes resíduos, por serem nutritivos, é empregada na alimentação animal, na forma de pellets.

Sem embargo, no geral, os subprodutos das frutas do género Citrus, evidenciam baixo valor comercial, por tanto, uma grande parte, é desperdiçada (GERHARDT, 2012), contribuindo aos milhões de toneladas de lixo urbano produzidos por este e outros restos de produtos agrícolas (STORCK, 2013).

Reduzir o desperdício de alimentos para enfrentar a fome no mundo e alcançar a segurança alimentar, é um dos objetivos da Agenda 2030 para o Desenvolvimento Sustentável das Nações Unidas (BRASIL, 2018).

Segundo Storck (2013), uma boa maneira de reduzir o desperdício de partes de alimentos vegetais ricos em nutrientes que são descartados, é o aproveitamento integral destes alimentos, transformando-os em ingredientes para novas receitas, iniciativa que pode enriquecer a culinária diária da população.

Por outro lado, Gerhardt (2012) demonstrou que as altas concentrações do ácido graxo poliinsaturado D-limoneno da casca de diversas frutas pertencentes ao gênero citrus, exerce uma ação antagônica sobre microorganismos responsáveis por diversas Doenças Transmitidas por Alimentos (DTAs). Este estudo sugere que a casca da laranja também possui esta característica e que por tanto, poderia ser utilizada como um conservante natural em produtos alimentícios.

Contudo, as fibras são consideradas bons substitutos de lipídios em diferentes formulações, desta maneira pelo alto teor de fibras presentes no albedo da laranja, a casca poderia ser utilizada como substituto da gordura na indústria da panificação (STOLL et al., 2015). Além disso, após análise do perfil bioquímico da farinha de casca de laranja, Storck et al. (2015) conclui que existe um alto teor de polifenóis, portanto é um subproduto que pode apresentar alta atividade antioxidante.

Levando em consideração que atualmente existe uma forte procura por alimentos funcionais, associada ao interesse de reduzir a perda de partes desprezíveis de alimentos agrícolas, este trabalho teve como objetivo desenvolver e avaliar sensorialmente formulações de bolos a base de aveia e fortificadas com farinha de casca de laranja e com farinha de casca e bagaço de laranja. 


\section{METODOLOGIA}

\subsection{ELABORAÇÃO DAS FARINHAS}

Para este trabalho, foram utilizadas cascas de laranja pera (Citrus sinensis $L$. Osbeck) prontas para consumo, adquiridas no comércio da cidade Cascavel - PR e produzidas em São Paulo - SP.

As frutas foram higienizadas com água corrente, colocadas em uma bacia na solução de $15 \mathrm{ml}$ de hipoclorito de sódio para $1 \mathrm{~L}$ de água, pelo período de 15 minutos e enxaguadas em água corrente. Posteriormente, as laranjas foram divididas em duas partes, sendo que de uma parte foi extraído somente o suco restando a casca e o bagaço e da outra parte foi retirada a fruta completa, deixado somente a casca. Ambas partes foram dispostas separadamente em travessas de vidro em estufa de circulação da marca Ethik Technology, modelo: $400-8 \mathrm{D} 200^{\circ} \mathrm{C}$, em temperatura de $60^{\circ} \mathrm{C}$ durante 35 horas. Logo, as cascas com e sem bagaço desidratadas foram trituradas separadamente em liquidificador, para posteriormente serem peneiradas e assim serem obtidas as farinhas de casca de laranja com e sem bagaço.

A seguir, na figura 01 encontra-se disposto o fluxograma da produção das farinhas de cascas de laranja e de casca de laranja e bagaço.

Higienização seguida de sanitização das laranjas em hipoclorito

Enxague em água corrente

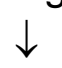

Extração do suco numa porção das laranjas

$\downarrow$

Secagem das cascas com e sem bagaço a $60^{\circ} \mathrm{C}$ por 35 horas em estufa de circulação

$\downarrow$

Trituração separada das cascas com bagaço e sem bagaço desidratas

Peneiração dos produtos finais

Obtenção das duas farinhas

Figura 01. Fluxograma de produção das farinhas de casca de laranja com e sem bagaço.

\subsection{DESENVOLVIMENTO DO PRODUTO}

Foram produzidas três formulações diferentes para o desenvolvimento das três amostras oferecidas na análise sensorial.

A formulação 1 (F1) corresponde ao bolo de laranja padrão sem adição de farinha de casca de laranja, produzido com os seguintes ingredientes: açúcar, farinha trigo, óleo de milho, ovos, fermento e suco de laranja. Primeiro, em uma bacia foram misturados o açúcar, a farinha e o fermento. Posteriormente foram adicionados os ovos, o óleo e o suco de laranja, obtendo-se uma massa homogênea. Finalmente, a mistura foi despejada numa travessa que foi levada ao forno em $160^{\circ} \mathrm{C}$ por 30 minutos. 
A formulação 2 (F2) corresponde ao bolo fortificado com farinha de casca de laranja. A formulação 3 (F3) representa o bolo fortificado com farinha de casca e bagaço de laranja. Para a produção de F2 e F3 foram utilizados os seguintes ingredientes: açúcar, farinha de aveia, farinha de casca de laranja para F2, farinha de casca e bagaço de laranja para F3, leite, óleo de milho, fermento e ovos. Primeiro, em uma bacia foram misturados o açúcar, o fermento, a farinha de aveia, a farinha de casca de laranja para F2 e a farinha de casca e bagaço de laranja para F3. Posteriormente foram adicionados os ovos e o óleo, obtendo-se uma massa homogênea, em cada preparação. Finalmente a mistura foi despejada em uma travessa que foi levada ao forno em $160^{\circ} \mathrm{C}$ por 30 minutos.

$\mathrm{Na}$ Tabela 01 segue o percentual de ingredientes utilizados nas três formulações.

Tabela 01. Percentual de ingredientes usados nas três formulações de bolos de laranja.

\begin{tabular}{lccc}
\hline \multicolumn{1}{c}{ Ingredientes } & $\mathrm{F} 1(\%)$ & $\mathrm{F} 2(\%)$ & $\mathrm{F} 3(\%)$ \\
\hline Açúcar & 13,5 & 18,9 & 18,9 \\
Aveia, farinha & 0,0 & 16,4 & 16,4 \\
Fermento químico & 0,9 & 1,1 & 1,1 \\
Farinha de casca de laranja & 0,0 & 0,4 & 0,0 \\
Farinha de casca e bagaço de laranja & 0,0 & 0,0 & 0,4 \\
Laranja, suco & 27,0 & 0,0 & 0,0 \\
Leite & 0,0 & 12,6 & 12,6 \\
Óleo de milho & 13,5 & 11,7 & 11,7 \\
Ovos & 18,0 & 38,9 & 38,9 \\
Trigo, farinha & 27,0 & 0,0 & 0,0 \\
\hline Total & 100 & 100 & 100
\end{tabular}

F1: formulação do bolo tradicional de laranja, F2: formulação do bolo de aveia fortificado com casca de laranja, F3: formulação do bolo de aveia fortificado com casca e bagaço de laranja.

A seguir, na figura 02 apresenta-se o fluxograma do desenvolvimento do produto.

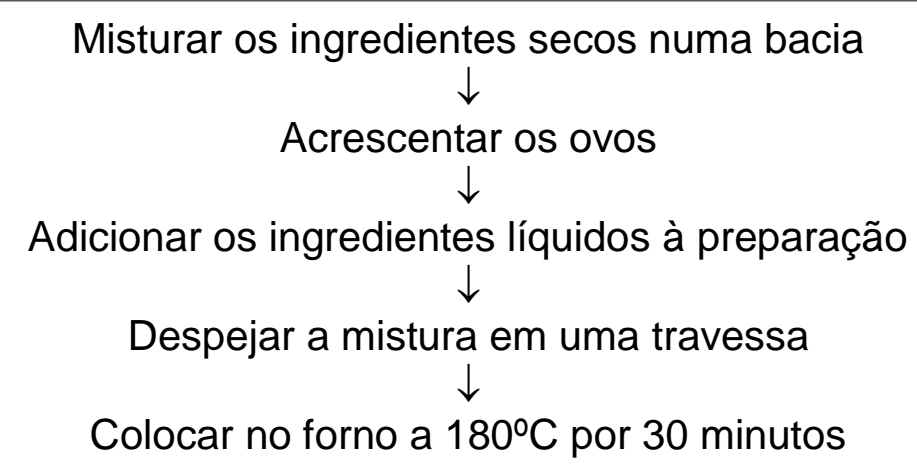

Figura 02. Fluxograma da elaboração das três formulações de bolos de laranja.

\subsection{ANÁLISE SENSORIAL}

A pesquisa foi realizada de acordo com os procedimentos de coleta de dados respeitando os critérios éticos, sendo que o projeto foi encaminhado ao comitê de ética e pesquisa com seres humanos e recebeu parecer favorável sob o $\mathrm{n}^{\circ} 2.078 .136$. Participaram da pesquisa indivíduos de ambos os sexos, com idades entre 18 a 50 
anos, sendo estes, estudantes e colaboradores de um Centro Universitário da cidade de Cascavel.

A análise sensorial foi realizada no laboratório de Nutrição em cabines específicas. Foram servidas três amostras de bolo de laranja, sendo a primeira amostra referente à formulação tradicional existente no mercado, a segunda amostra à base de farinha de aveia e fortificada com farinha de casca de laranja e a terceira à base de farinha de aveia fortificada com farinha de casca e bagaço de laranja. As amostras foram servidas de forma aleatória em copos codificados com algarismos de 3 dígitos e com quantidades padronizadas de aproximadamente $15 \mathrm{~g}$ cada uma, não havendo repetição da amostra. Também foi disponibilizado um copo de água nos intervalos da amostra para limpeza do palato.

A aceitabilidade das amostras foi avaliada utilizando-se uma escala hedônica estruturada de nove pontos, cujos extremos de notas variavam de gostei muitíssimo (9) à desgostei muitíssimo (1). Foram avaliados atributos de aceitação global, aparência, aroma, sabor e textura. Também foi avaliada a intenção de compra do consumidor por meio de uma escala estruturada de cinco pontos cujos extremos variavam de certamente compraria (5), à certamente não compraria (1) (DUTCOSKY, 2013).

Os resultados da análise sensorial foram avaliados por meio da análise de variância (ANOVA), e as médias foram testadas pelo teste de Tukey considerando um nível de significância de 5\%. Para a análise estatística utilizou-se o programa Microsoft Excel 2010.

\subsection{CÁLCULO NUTRICIONAL}

O valor nutricional das formulações desenvolvidas foi determinado segundo os critérios estabelecidos na Resolução RDC n³60, de 23 de dezembro de 2003, regulamento técnico sobre rotulagem nutricional de alimentos embalados. (BRASIL, 2003). O cálculo nutricional foi obtido por meio da consulta na tabela de informação nutricional Tabela Brasileira de Composição de Alimentos - TACO (UNICAMP, 2011), e baseados em estudos realizados por Storck (2013) e Stoll (2015).

\section{RESULTADOS E DISCUSSÃO}

Após terminado o processo de desidratação das cascas de laranjas utilizadas, observou-se uma redução no peso indicando um percentual de umidade de $78,3 \%$ nas cascas com bagaço e de $72,7 \%$ nas cascas sem o bagaço. Na Tabela 02 se apresentam os teores de macronutrientes, fibra e sódio presentes nas três formulações.

Com base na análise da composição nutricional da farinha de casca de laranja, produzida em condições similares às empregadas no presente trabalho, realizada por Storck (2013), verifica-se que em $100 \mathrm{~g}$ de farinha de casca de laranja há: $83 \mathrm{kcal} ; 18$ $\mathrm{g}$ de carboidratos; 1,8 $\mathrm{g}$ de proteínas; 0,5 g de lipídios; 3,6 g de fibras e $631 \mathrm{mg}$ de polifenóis. Assim, destaca-se o alto conteúdo deste último componente orgânico na farinha, pois quando comparado ao teor de polifenóis referente a $390 \mathrm{mg}$ encontrados em $100 \mathrm{~g}$ de amostra de videiras destinadas à produção de vinhos analisadas por Abe et al. (2007), a farinha de casca de laranja quase duplica o conteúdo polifenólico em $100 \mathrm{~g}$ de produto, por tanto pode-se classificar a farinha de casca de laranja como um potente antioxidante. 
Tabela 02. Composição nutricional de uma porção de $100 \mathrm{~g}$ de produto desenvolvido com as três formulações de bolos

\begin{tabular}{lcccccc}
\hline & \multicolumn{2}{c}{ F1 } & \multicolumn{2}{c}{ F2 } & \multicolumn{2}{c}{ F3 } \\
& Quantidade & $\% \mathrm{VD}\left({ }^{*}\right)$ & Quantidade & $\% \mathrm{VD}\left({ }^{*}\right)$ & Quantidade & $\% \mathrm{VD}\left(^{*}\right)$ \\
\hline VCT & $304 \mathrm{Kcal}$ & 15,2 & $308 \mathrm{Kcal}$ & 15,4 & $308 \mathrm{Kcal}$ & 15,4 \\
CHO & $36,4 \mathrm{~g}$ & 12,1 & $32,2 \mathrm{~g}$ & 10,7 & $32,2 \mathrm{~g}$ & 10,7 \\
PTN & $5,2 \mathrm{~g}$ & 6,9 & $7,9 \mathrm{~g}$ & 10,5 & $7,9 \mathrm{~g}$ & 10,5 \\
LIP & $15,5 \mathrm{~g}$ & 28,2 & $16,9 \mathrm{~g}$ & 30,8 & $16,9 \mathrm{~g}$ & 30,8 \\
FB & $0,63 \mathrm{~g}$ & 2,5 & $1,8 \mathrm{~g}$ & 6,6 & $1,8 \mathrm{~g}$ & 7,1 \\
$\mathrm{Na}$ & $123 \mathrm{mg}$ & 5,1 & $186 \mathrm{mg}$ & 7,8 & $186 \mathrm{mg}$ & 7,8 \\
\hline
\end{tabular}

$\left({ }^{*}\right) \%$ Valores Diários (VD), são expressos com base em uma dieta de 2.000kcal. F1: formulação do bolo tradicional de laranja, F2: formulação do bolo de aveia fortificado com casca de laranja, F3: formulação do bolo de aveia fortificado com casca e bagaço de laranja. VCT: valor calórico total, CHO: carboidratos, PNT: proteínas, LIP: lipídios totais, FB: fibras, Na: sódio.

Quanto à superioridade no teor de fibras de F2 e F3 em relação a F1, como descreve a Tabela 02, verificou-se que a farinha de aveia foi responsável por esta característica. Gutkoski et al. (2009), afirma que a viscosidade da massa em uma formulação de bolo, depende da concentração de aveia e gordura da mesma, assim, existe uma relação inversamente proporcional entre a fração desses ingredientes, onde, quanto maior a quantidade de aveia e menor a de gordura, maior é a porosidade do bolo. Por outro lado, Segundo Stoll (2015), se a fibra utilizada em alguma preparação culinária está presente em uma proporção acima de $2,5 \%$ do total dos ingredientes, obtém-se um alimento rico em fibras e podendo ser isento de gorduras, pois a fibra tem a capacidade de substituir a adição de gordura na preparação.

Segundo Bublitz (2013) a análise centesimal demonstrou o teor de fibra bruta de farinha de albedo da laranja $(16,20 \mathrm{~g} / 100 \mathrm{~g})$ cerca de cinco vezes maior quando comparada com uma farinha tradicional de trigo, por isso pode ser considerado um alimento funcional. De acordo com a Resolução n¹8, de 30 de abril de 1999 da Agência Nacional de Vigilância Sanitária (BRASIL, 1999), a alegação de propriedade funcional é relativa ao papel metabólico ou fisiológico que o nutriente ou não nutriente tem no crescimento, desenvolvimento, manutenção e outras funções normais do organismo humano.

Enquanto ao teor de óleos essenciais presentes na casa dos cítricos, estudos demonstram que o conteúdo destes óleos corresponde, maioritariamente, ao óleo insaturado D-limoneno, que apresenta uma ação inibitória sobre o crescimento de microrganismos, especificamente sobre aqueles que provocam DTAs (GERHARDT, 2012). Desta maneira, pode-se inferir que a casca de laranja, por ser um cítrico, também dificultaria a sobrevida desse tipo de microrganismos, podendo ter um potencial de uso na indústria de alimentos como um conservante natural.

Em relação à análise sensorial realizada, na Tabela 03 encontram-se expostas as médias dos resultados para cada atributo avaliado. Sendo assim, verifica-se que houve diferença estatística significativa $(p<0,05)$ na aceitação de todos os atributos de $F 1$ em relação a F2 e F3, que não diferiram estatisticamente $(p>0,05)$ entre si. Apesar deste fato, de acordo com a escala hedônica utilizada, os provadores aprovaram F2 e F3 com notas que variaram entre 6 e 7 , sendo que a primeira correspondia a "gostei moderadamente" e a segunda a "gostei ligeiramente".

É possível que F1 teve uma melhor aceitação porque a farinha utilizada como base na receita foi de trigo, diferentemente das receitas de F2 e F3, onde a farinha base foi de aveia. Este fator interferiu em todos os atributos sensoriais, favorecendo 
principalmente a aparência e textura de F1. Porém, do ponto de vista nutricional F2 e F3 apresentam melhores características, destacando o baixo teor de carboidratos simples, já que na sua composição não teve adição de suco de laranja para dar os atributos característicos de aroma e sabor de um bolo de laranja, ao contrário de F1, onde foi o suco o ingrediente que forneceu essas propriedades, acrescentando maior concentração de carboidrato simples ao produto.

Em relação à aceitação de $\mathrm{F} 2$ e F3, como descrito na Tabela 3, não houve diferença estatística ( $p>0,05$ ) entre elas. Contudo, F3 foi a formulação que recebeu as menores notas designadas por parte dos provadores, principalmente em relação ao sabor, isto devido a que na sua preparação havia presença do bagaço da fruta que Ihe entregou um sabor mais amargo.

Tabela 03. Resultado da análise estatística realizada nas médias encontradas para cada atributo avaliado na análise sensorial dos bolos de laranja

\begin{tabular}{lcccc}
\hline \multicolumn{1}{c}{ Atributos } & $\mathrm{F} 1$ & $\mathrm{~F} 2$ & $\mathrm{~F} 3$ & Valor $\mathrm{p}$ \\
\hline Aceitação global & $8.1 \pm 1.2^{\mathrm{a}}$ & $6.9 \pm 1.4^{\mathrm{b}}$ & $6.4 \pm 1.8^{\mathrm{b}}$ & $<0,0001$ \\
Aparência & $8.1 \pm 1.0^{\mathrm{a}}$ & $6.8 \pm 1.6^{\mathrm{b}}$ & $6.0 \pm 1.9^{\mathrm{b}}$ & $<0,0001$ \\
Aroma & $8.1 \pm 1.3^{\mathrm{a}}$ & $7.1 \pm 2.0^{\mathrm{b}}$ & $6.3 \pm 1.8^{\mathrm{b}}$ & $<0,0001$ \\
Sabor & $8.4 \pm 1.0^{\mathrm{a}}$ & $7.0 \pm 1.9^{\mathrm{b}}$ & $6.1 \pm 2.3^{\mathrm{b}}$ & $<0,0001$ \\
Textura & $8.3 \pm 0.9^{\mathrm{a}}$ & $6.8 \pm 2.0^{\mathrm{b}}$ & $6.2 \pm 2.2^{\mathrm{b}}$ & $<0,0001$ \\
Intenção de compra & $4.6 \pm 0.6^{\mathrm{a}}$ & $3.5 \pm 1.0^{\mathrm{b}}$ & $3.2 \pm 1.2^{\mathrm{b}}$ & $<0,0001$ \\
\hline
\end{tabular}

A médias seguidas de letras diferentes diferiram estatisticamente no teste de Tukey $(p<0,005)$.

\section{CONCLUSÃO}

Foi possível desenvolver um produto alimentício rico em polifenóis e fibras por meio do aproveitamento das cascas de laranjas que normalmente são descartadas na produção do suco desta fruta, fato que contribui à diminuição da quantidade de lixo produzido por esta indústria e ao enriquecimento nutricional de preparações mediante o aproveitamento integral de alimentos vegetais. Dessa maneira, tanto a aveia como a casca de laranja, apresentam características funcionais que podem enriquecer preparações nutricionalmente, porém a palatabilidade de preparações com ingredientes tradicionais se reflete em uma melhor aceitação nos consumidores.

Contudo é necessário aprimorar as formulações para melhorar a aceitação do produto, principalmente no tocante, por um lado, à substituição da gordura pela fibra presente, tanto na farinha de casca de laranja como na aveia e por outro lado à utilização somente da casca da fruta, pois o bagaço outorga um sabor amargo à preparação.

\section{REFERÊNCIAS}

ABE, L.T.; DA MOTA, R.V.; LAJOLO, F.M.; GENOVESE, M.I. Compostos fenólicos e capacidade antioxidante de cultivares de uvas Vitis labrusca L. e Vitis vinifera L. Ciênc. Tecnol. Aliment., Campinas, 27(2): 394-400, abr.-jun. 2007.

BUBLITZ, S., EMMANOUILIDIS, P., OLIVEIRA, M. S. R. DE, ROHLFES, A. L. B., BACCAR, N.M., CORBELLINI, V. A., MARQUARDT, L. Produção de uma farinha de albedo de laranja como forma de aproveitamento de resíduo. Revista Jovens Pesquisadores, Santa Cruz do Sul, v. 3, n. 2, p. 112-121, 2013. 
BRASIL. Centro de Estudos e Debates Estratégicos. Perdas e desperdício de alimentos. Estratégias para redução. Consultoria Legislativa. Brasília - DF, 2018.

BRASIL. Ministério da Saúde. Agência Nacional de Vigilância Sanitária. Resolução no 19, de 30 de abril de 1999. Regulamento técnico de procedimentos para registro de alimento com alegação de propriedades funcionais e ou de saúde em sua rotulagem. Diário Oficial da República Federativa do Brasil, Brasília - DF, 1999.

BRASIL. Ministério de Agricultura, Pecuária e Abastecimento e Empresa Brasileira de Pesquisa Agropecuária (EMBRAPA). A laranja e seus subprodutos na alimentação animal. Embrapa Tabuleiros Costeiros, Aracaju - SE, 2001.

BRASIL. Ministério de Agricultura, Pecuária e Abastecimento Sumário Executivo Complexo Laranja. Departamento de Crédito e Estudos Econômicos, 2018.

BRASIL, Ministério da Saúde. Agência Nacional de Vigilância Sanitária - ANVISA. Resolução - RDC № 360, de 23 de dezembro de 2003. Brasília - DF, 2003.

CYPRIANO, D.Z.; SILVA, L.L.; MARIÑO, M.A.; TASIC, L. A Biomassa da Laranja e seus Subprodutos. Revista Virtual Quim., 9 (1), 176-191, 2017.

DUTCOSKY, S.D. Análise sensorial de alimentos. $4^{a}$ Ed. Editora Champagnat. Curitiba - PR, 2013.

FERNANDES, I.J.; KIELING, A.G.; BREHM, F.A.; AGOSTI, A.; MORAES, C.A.M. Avaliação da Extração de Óleo Essencial do Resíduo Casca de Laranja. 4 Fórum Internacional de Resíduos Sólidos. Porto Alegre - RS, 2013.

FRANCO, A.S.M. O suco de laranja brasileiro no mercado global. Instituto Paranaense de Desenvolvimento Econômico e Social (IPARDES). Análise conjuntural, v.38, n.11-12, 2016.

GERHARDT, C.; WIEST, J.M.; GIROLOMETTO, G.; SILVA, M.A.S.; WESCHENFELDER, S. Aproveitamento da casca de citros na perspectiva de alimentos: prospecção da atividade antibacteriana. Brazilian Journal Food Technology., IV SSA, p. 11-17, 2012.

GUTKOSKI, L.C.; TEIXEIRA, D.M.F.; DURIGON, A.; GANZER, A.G.; BERTOLIN, T.E.; COLLA, L.M. Influência dos teores de aveia e de gordura nas características tecnológicas e funcionais de bolos. Ciênc. Tecnol. Aliment., Campinas, 29(2): 254261, abr.-jun. 2009.

LIMA, U.A. Matérias-primas dos Alimentos. $1^{\circ}$ Ed. Editora Edgard Blucher. São Paulo - SP, 2010.

SANTANA, M.F.S.; GASPARETTO, C.A. Microestrutura da fibra alimentar do albedo de laranja: um estudo por técnicas físicas e análise de imagens. Ciênc. Tecnol. Aliment., Campinas, 29(1): 124-134, jan.-mar. 2009. 
STOLL, L.; FLÔRES, S.H.; THYS, R.C.S. Fibra de casca de laranja como substituto de gordura em pão de forma. Revista Ciência Rural, Santa Maria, v.45, p.567-573, 2015.

STORCK, C. R.; NUNES, G. L.; OLIVEIRA, B. B.; BASSO, C. Folhas, talos, cascas e sementes de vegetais: composição nutricional, aproveitamento na alimentação e análise sensorial de preparações. Revista Ciência Rural, Santa Maria, v.43. n.3, p.537-543, 2013.

UNIVERSIDADE ESTADUAL DE CAMPINAS - UNICAMP. Tabela brasileira de composição de alimentos - TACO. 4a Ed. rev. e ampl. Campinas: UNICAMP/NEPA, 2011. 